\title{
Tin Tungstate Nanoparticles: A Photosensitizer for Photodynamic Tumor Therapy
}

Carmen Seidl $^{\dagger}$, Jan Ungelenk ${ }^{\star}$, Eva Zittel $^{\dagger}$, Thomas Bergfeldt ${ }^{\S}$, Jonathan P. Sleeman ${ }^{\perp}$, Ute Schepers ${ }^{\dagger *}$, and Claus Feldmann ${ }^{* *}$

†Institute of Toxicology and Genetics, Karlsruhe Institute of Technology (KIT), Hermann-vonHelmholtz-Platz 1, 76344 Eggenstein-Leopoldshafen (Germany), E-mail: ute.schepers@kit.edu

\#nstitute of Inorganic Chemistry, Karlsruhe Institute of Technology (KIT), Engesserstr. 15, 76131 Karlsruhe (Germany), E-mail: claus.feldmann@,kit.edu

${ }^{\S}$ Institute of Applied Materials Physics, Karlsruhe Institute of Technology (KIT), Hermann-vonHelmholtz-Platz 1, 76344 Eggenstein-Leopoldshafen (Germany)

${ }^{\perp}$ Medical Faculty Mannheim of the University of Heidelberg, Centre for Biomedicine and Medical Technology Mannheim (CBTM), Ludolf-Krehl-Str. 13-17, 68167 Mannheim (Germany)

\section{- SUPPORTING INFORMATION -}

\section{Content}

1. Analytical tools

2. Synthesis of protamine-functionalized $\beta-\mathrm{SnWO}_{4}$ nanoparticles

3. In vivo tests of $\beta-\mathrm{SnWO}_{4}$ nanoparticles with orthotopic breast cancer BALB/c mouse model

4. Blood parameters

5. Haematoxylin- and eosine-staining (progressive)

References 


\section{Analytical tools}

Scanning electron microscopy (SEM) was carried out with a Zeiss Supra 40 VP microscope. Diluted aqueous suspensions of as-prepared $\beta$-SnWO $\mathrm{SO}_{4}$ were deposited on silicon wafers and evaporated. The acceleration voltage was in the range of $5-10 \mathrm{kV}$ and the working distance was $3 \mathrm{~mm}$.

Transmission electron microscopy (TEM): Transmission electron microscopy (TEM) was conducted with a Philips CM200-FEG microscope operating at $200 \mathrm{kV}$. TEM samples were prepared by evaporating suspensions in ethanol on amorphous carbon (Lacey-)film suspended on copper grids. The deposition of the samples on the carbon (Lacey-)film copper grids was performed under Argon atmosphere in a glovebox.

Dynamic light scattering (DLS) was conducted with polystyrene cuvettes applying a Nanosizer ZS from Malvern Instruments.

Zeta-potential measurements were performed in polystyrene cuvettes applying a Nanosizer ZS from Malvern Instruments. To this purpose, aqueous suspensions were titrated from neutral to alkaline and acidic $\mathrm{pH}$ by addition of $\mathrm{NaOH}$ and $\mathrm{HCl}$, respectively.

A blue-LED light source (LED lenser) was purchased by Zweibrüder Optoelectronics. The blue-light LED exhibited a total length of $66 \mathrm{~mm}$, a diameter of $14 \mathrm{~mm}$ and a total weight of $19 \mathrm{~g}$. It operated with a luminous flux of $12 \mathrm{~lm}$ and an irradiance of $0.2 \mathrm{~W} / \mathrm{cm}^{2}$ at a wavelength range of 440-500 $\mathrm{nm}$ and with $\lambda_{\max }=465 \mathrm{~nm}$ (cf. main text: Figure $\left.2 a\right)$.

Diffuse reflectance spectra (UV-VIS) of powders were recorded in a wavelength interval of 250$800 \mathrm{~nm}$ with a Varian Cary 100 spectrometer, equipped with an integrating sphere against $\mathrm{BaSO}_{4}$ as a reference.

Fourier-transform infrared spectroscopy (FT-IR) was performed on a Bruker Vertex 70 FT-IR spectrometer using $\mathrm{KBr}$ pellets.

Differential thermal analysis and thermogravimetry $(D T A / T G)$ were performed with a Netzsch STA 449 F3 applying $\alpha-\mathrm{Al}_{2} \mathrm{O}_{3}$ as crucible material and reference sample. The samples were heated under $\mathrm{N}_{2}$ flow to $800{ }^{\circ} \mathrm{C}$ with a heating rate of $1 \mathrm{~K}$ minute ${ }^{-1}$. 


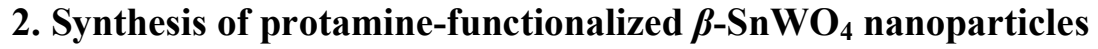

Details regarding the synthesis were already reported elsewhere. ${ }^{1}$ According to a typical recipe, $\mathrm{SnCl}_{2}$ (417.1 mg, $2.2 \mathrm{mmol}$ ) was dissolved in $2.5 \mathrm{~mL}$ of carbonated water. Under vigorous magnetic stirring, this solution was added to $2.5 \mathrm{~mL}$ of an equimolar aqueous solution of $\mathrm{Na}_{2} \mathrm{WO}_{4} \times 2 \mathrm{H}_{2} \mathrm{O}(725.7 \mathrm{mg})$ at ambient temperature. A bright yellow nanomaterial was generated immediately and the resulting suspension can be directly diluted with $20 \mathrm{~mL}$ of phosphate-buffered saline (PBS: $8.0 \mathrm{~g} / \mathrm{L} \mathrm{NaCl} ; 0.2 \mathrm{~g} / \mathrm{L} \mathrm{KCl}$; $1.42 \mathrm{~g} / \mathrm{L} \mathrm{Na}_{2} \mathrm{HPO}_{4} ; 1.78 \mathrm{~g} / \mathrm{L} \mathrm{Na}_{2} \mathrm{HPO}_{4} \cdot 2 \mathrm{H}_{2} \mathrm{O} ; 0.27 \mathrm{~g} / \mathrm{L} \mathrm{KH}_{2} \mathrm{PO}_{4}$ ) (Figure S1). The obtained nanomaterial was collected by centrifugation $(15 \mathrm{~min}, 25,000 \mathrm{rpm})$, redispersed two times in PBS and centrifuged again for washing. Finally, the washed suspension was centrifuged for 2 minutes at 10,000 rpm in order to remove agglomerates. The obtained centrifugate represents an aqueous suspension of $\beta-\mathrm{SnWO}_{4}$ nanoparticles showing diameters well below $20 \mathrm{~nm}$ and a mean diameter of $8( \pm 2) \mathrm{nm}(0.13 \mathrm{wt}-\%, 45 \%$ yield of dry powder) being fairly stable without any additional stabilizing agents (cf. main text: Figure 1).

To improve membrane penetration and cell uptake, the as-prepared $\beta$-SnWO 4 nanoparticles were coated with protamine. ${ }^{2}$ To this concern, protamine solution (protamine sulfate from herring) was added dropby-drop to the above described PBS suspension of the $\beta$-SnWO $\mathrm{Sn}_{4}$ nanoparticles in order to electrostatically bind the protamine to the particle surface (Figure S1). As a cationic biopolymer protamine adheres easily on the negatively charged $\beta-\mathrm{SnWO}_{4}$ surface and naturally reduces the sum zeta potential to about $-20 \mathrm{mV}$ (cf. main text: Figure 1c). Note that only biocompatible solvents were used throughout the nanoparticle synthesis with PBS having the same $\mathrm{pH}(\mathrm{pH}=7.4)$ and the same osmotic pressure than human blood.

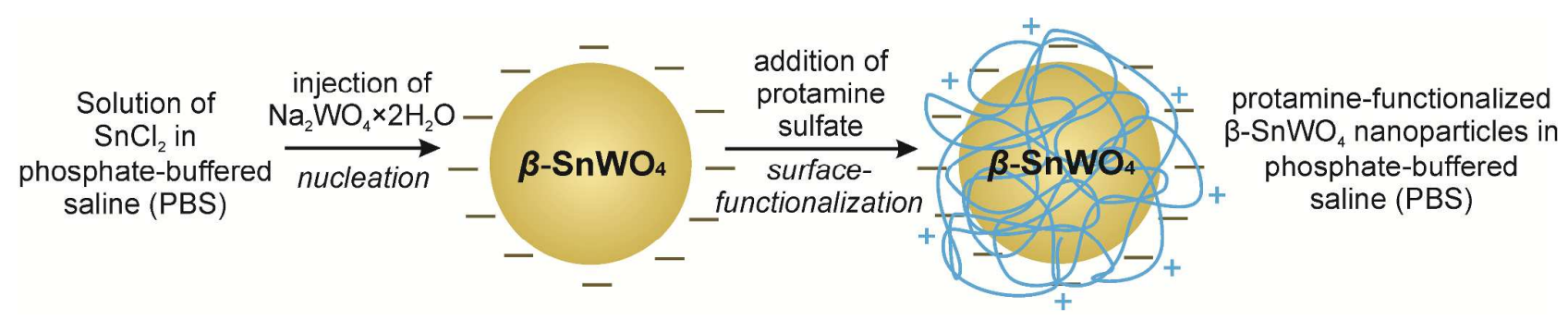

Figure S1. Scheme illustrating the water-based synthesis of $\beta$-SnWO $\mathrm{O}_{4}$ nanoparticles as well as the subsequent protamine functionalization.

The presence of protamine as a surface capping on the particle surface was validated by FT-IR spectra (Figure S2). While comparing to pure protamine as a reference, all characteristic vibrations $(v(\mathrm{O}-\mathrm{H})$ : 3600-3000; $v(\mathrm{C}-\mathrm{H}): 2950-2800 ; v(\mathrm{C}=\mathrm{O}): 1700-1500$; fingerprint area: 1500-500) can be identified for the protamine-functionalized $\beta-\mathrm{SnWO}_{4}$ nanoparticles as well. Thermogravimetry indicated the amount of protamine on the $\beta$-SnWO $\mathrm{W}_{4}$ nanoparticles (Figure S3). Accordingly, a first weight loss (6\%) between 
room temperature and $120^{\circ} \mathrm{C}$ can be ascribed to an evaporation of water adhered on the particle surface. As a next step, a loss of about $34 \%$ by mass in a temperature range of $220-500{ }^{\circ} \mathrm{C}$ is related to the thermal decomposition of protamine. In view of the particle size $(8 \pm 2 \mathrm{~nm})$ of the as-prepared $\beta$-SnWO $\mathrm{Sn}_{4}$ nanoparticles, such a load of 30-35\% is very typical for a protamine-type surface-capping. Non-capped asprepared $\beta-\mathrm{SnWO}_{4}$ nanoparticles as a reference show a similar evaporation of water, but no weight loss in the temperature range of $220-500{ }^{\circ} \mathrm{C}$. The slight increase (about $4 \%$ ) of mass at $300-350{ }^{\circ} \mathrm{C}$ is due to the oxidation of $\mathrm{Sn}^{2+}$ to $\mathrm{Sn}^{4+}$ in air according to the following reaction: $\mathrm{SnWO}_{4}+1 / 2 \mathrm{O}_{2} \rightarrow \mathrm{SnO}_{2}+\mathrm{WO}_{3}$ (calculated: $+4.4 \%)$.

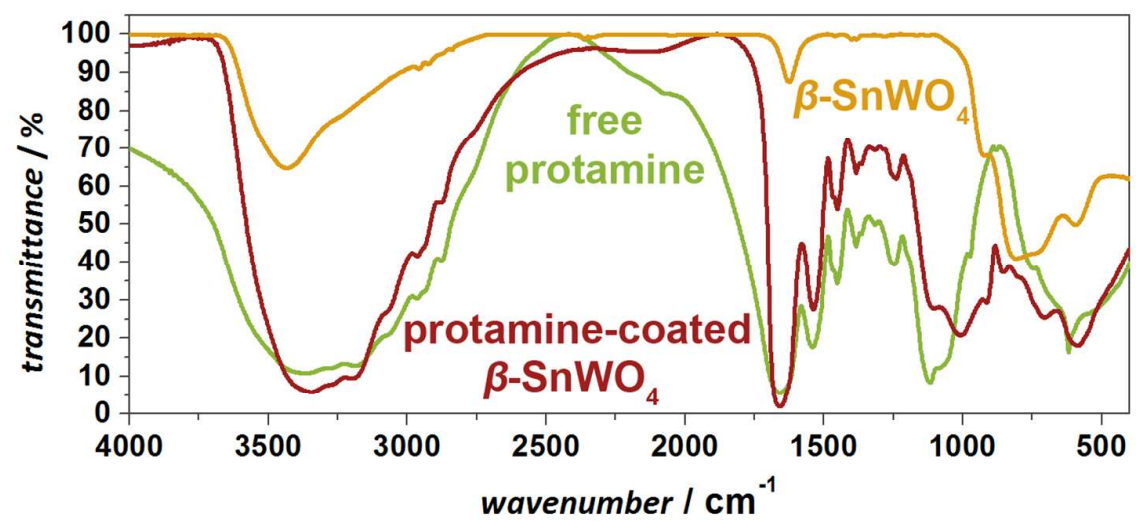

Figure S2. FT-IR spectrum of the protamine-functionalized $\beta$-SnWO $\mathrm{O}_{4}$ nanoparticles (as-prepared $\beta$-Sn $\mathrm{WO}_{4}$ nanoparticles and pure protamine as a reference).

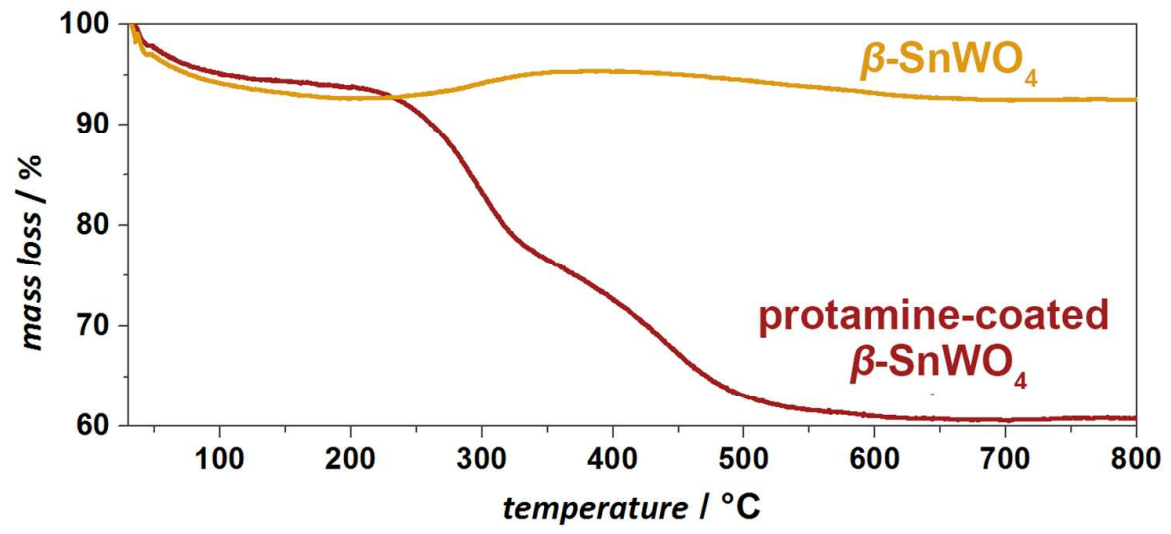

Figure S3. Thermogravimetry of the protamine-functionalized $\beta$-SnWO $\mathrm{O}_{4}$ nanoparticles (asprepared $\beta$-SnWO $\mathrm{W}_{4}$ nanoparticles as a reference).

\section{In vivo tests of $\beta$-SnWO $\mathrm{S}_{4}$ nanoparticles with orthotopic breast cancer BALB/c mouse model}

4T1 cells were maintained in DMEM medium supplemented with $10 \%$ FCS and 1\% penicillin/streptomycin at $37{ }^{\circ} \mathrm{C}$ in a humidified incubator with $5 \% \mathrm{CO}_{2} \cdot 1 \cdot 10^{6}$ cells in $100 \mu 1$ of PBS were in- 
jected orthotopically into the mammary fat pad of 8-12 weeks old female BALB/c mice. Before injection, cells were tested for mycoplasma with the VenorGeM ${ }^{\circledR}$ Mycoplasma Detection Kit. 11 days after tumor induction, mice were randomized into three experimental groups (six mice per group). Mice of group 1 were treated intraperitoneally with protamine-functionalized $\beta-\mathrm{SnWO}_{4}$ nanoparticles $(23 \mathrm{mg} / \mathrm{kg}$ of body weight) trice a week for a total of seven doses. Subsequently, the tumor of mice from group 1 was illuminated locally with a blue-LED point-light source $\left(\lambda_{\max }=465 \mathrm{~nm}\right)$ for 5 minutes. Control groups were treated with PBS (group 3) or doxorubicin (group 2, $2.5 \mathrm{mg} / \mathrm{kg}$ of body weight). Animals were killed and an autopsy was performed when they became moribund or when tumors grew to the German legal limit.

The tumor growth was monitored by measuring the tumor diameters with calipers twice a week. The tumor volume was calculated using the formula $4 / 3 \cdot \pi \cdot a \cdot b \cdot c$ (ellipsoid). The treatment-related toxicity was determined by mouse weights weekly. Before the end of the study, 26 days after tumor induction, blood was collected via cardiocentesis. Tumor, inguinal and auxiliary lymph nodes were dissected, weighed and measured in size. The lung was macroscopically examined for the occurrence of metastases. Lung, tumor and lymph nodes were fixed overnight in 3.7\% paraformaldehyd, embedded in paraffin and cut into sections for histological staining with hematoxylin and eosin. Animal procedures were approved by the regional government on animal experimentation in Germany: 35-9185.81/G-5/10.

Figure S4 and Figure S5 show the body weight and tumor growth of all animals as documented during the complete survey. The body weight for group 1 ( $\beta$-SnWO 4 -treated), group 2 (doxorubicin-treated) and group 3 (DPBS-treated) is rather constant during the complete survey. Slight increase of weight can be attributed to tumor growth.
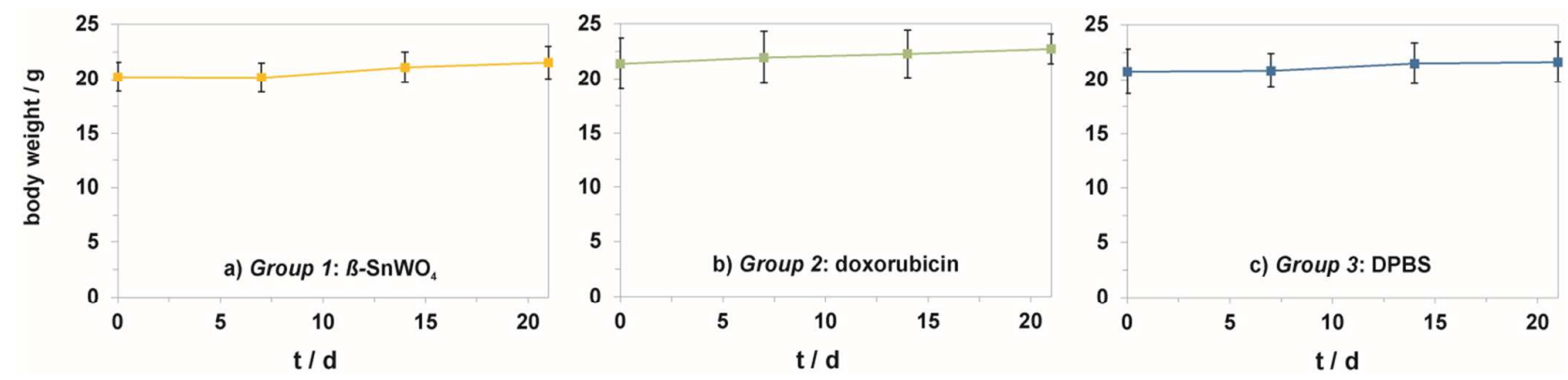

Figure S4. Body weight of tumor-bearing BALB/c-mice: a) Group 1-treated with $\beta$-SnWO ${ }_{4}$ nanoparticles (23 mg/kg of body weight) and subsequently illuminated for 5 minutes with blue-light LED $\left(\lambda_{\max }=465 \mathrm{~nm}\right)$ at the site of the tumor; b) Group 2-treated with doxorubicin $(2.5 \mathrm{mg} / \mathrm{kg} \mathrm{of}$ body weight); c) Group 3-treated with DPBS (each value represents the mean of $n=6$ animals in the respective group). 
The tumor size of mice treated with physiological saline (DPBS, group 3) and doxorubicin (group 2) increased from day 1 to day 21 (Figure S5; cf. main text: Figure 6). In both cases some tumors grew to volumes up to $6 \mathrm{~cm}^{3}$ (Figure S5; cf. main text: Figure 6). Moreover, only the control group and the doxorubicin-treated group showed cortically visible, broken-up tumors. As broken-up tumors continuously lose blood, they appear smaller than non-broken-up tumors. This explains the higher statistical spreading of the tumor volume of individual animals of the DPBS (group 3) and doxorubicin group (group 2). In contrast, $\beta$-SnWO 4 -treated mice (group 1 ) show a fundamentally reduced growth of the primary tumor (Figure S5; cf. main text: Figure 6). Moreover, all primary tumors remain at tumor volumes below $3 \mathrm{~cm}^{3}$ and broken-up tumors were not observed for $\beta$-SnWO $\mathrm{S}_{4}$-treated mice (group 1). Consequently, the statistical spreading between the different animals in this group is much narrower as compared to mice treated with physiological saline (DPBS, group 3) and doxorubicin (group 2) (Figure S5; cf. main text: Figure 6).
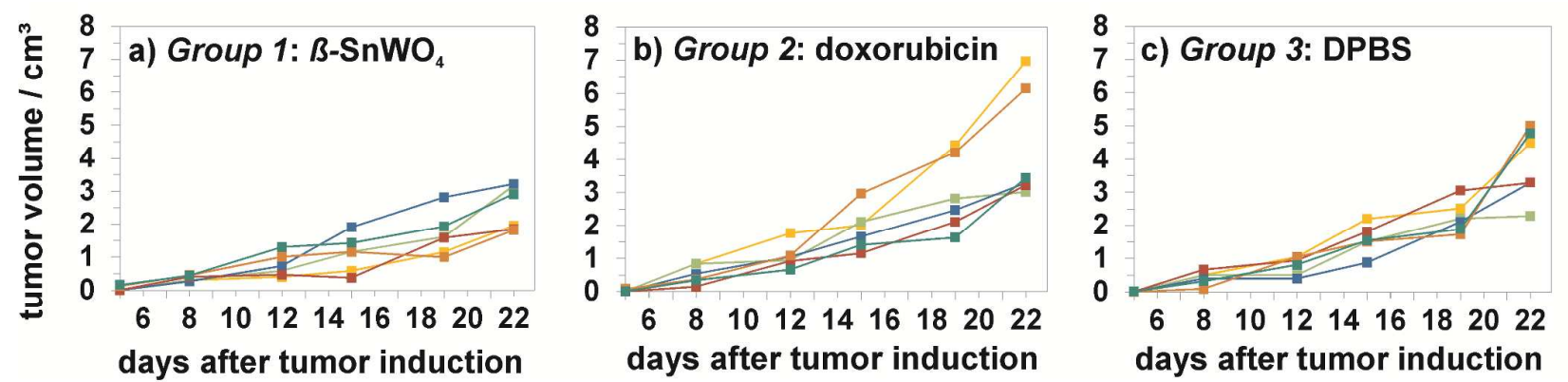

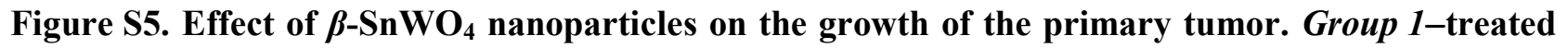
with $\beta$-SnWO $\mathrm{S}_{4}$ nanoparticles ( $23 \mathrm{mg} / \mathrm{kg}$ of body weight) and subsequently illuminated for 5 minutes with blue-light LED $\left(\lambda_{\max }=465 \mathrm{~nm}\right)$ at the site of the tumor; Group 2-treated with doxorubicin (2.5 mg/kg of body weight); Group 3-treated with DPBS (each value represents the mean of $n=6$ animals in the respective group). It is to be noted that broken-up tumors in the DPBS and doxorubicin group continuously lose blood and appear smaller than non-broken-up tumors, leading to certain spreading of the measured tumor volume.

In addition to the $\beta$-SnWO 4 -treated mice (group 1) as well as the doxorubicine-treated mice (positive control, group 2) and DPBS-treated mice (negative control, group 3), the $\beta$ - $\mathrm{SnWO}_{4}$-treated mice were, on the one hand, illuminated with a blue-light LED for 5 minutes at the site of the tumor (group 1, with bluelight LED illumination), and on the other hand, not illuminated with a blue-light LED (group 1, without blue-light LED illumination). While the data of mice treated with $\beta-\mathrm{SnWO}_{4}$ nanoparticles with subsequent blue-light LED illumination were already discussed in the main text (cf. main text: Figures 6-9), data of mice treated with $\beta-\mathrm{SnWO}_{4}$ nanoparticles but without blue-light LED illumination are shown in Figures S6-S8. 


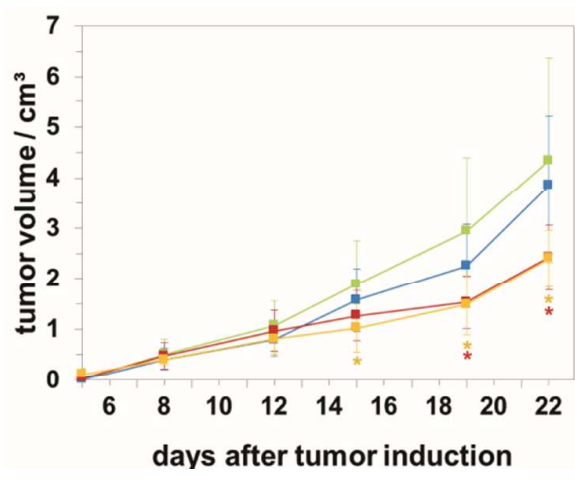

- doxorubicin (group 2)

- DPBS (group 3*)

- $\beta-\mathrm{SnWO}_{4}$ (group 1, without blue-light LED illumination)

" $\beta$-SnWO (group 1, with blue-light LED illumination)

Figure S6. Effect of $\beta$-SnWO $\mathrm{W}_{4}$ nanoparticles on the growth of the primary tumor (cf. main text: Figure 6): Group 1-treated with $\beta$-SnWO 4 nanoparticles $(23 \mathrm{mg} / \mathrm{kg}$ of body weight) with and without blue-light LED illumination; Group 2-treated with doxorubicin (2.5 mg/kg of body weight); Group 3-treated with DPBS (each value represents the mean of $n=6$ animals in the respective group; SI: Figure S5 for detailed data on individual animals). Significance between the respective groups was determined according to student's $t$-test with $p<0.05$. (*) It is to be noted that brokenup tumors in the DPBS and doxorubicin group continuously lose blood and appear smaller than non-broken-up tumors, leading to certain spreading of the measured tumor volume.

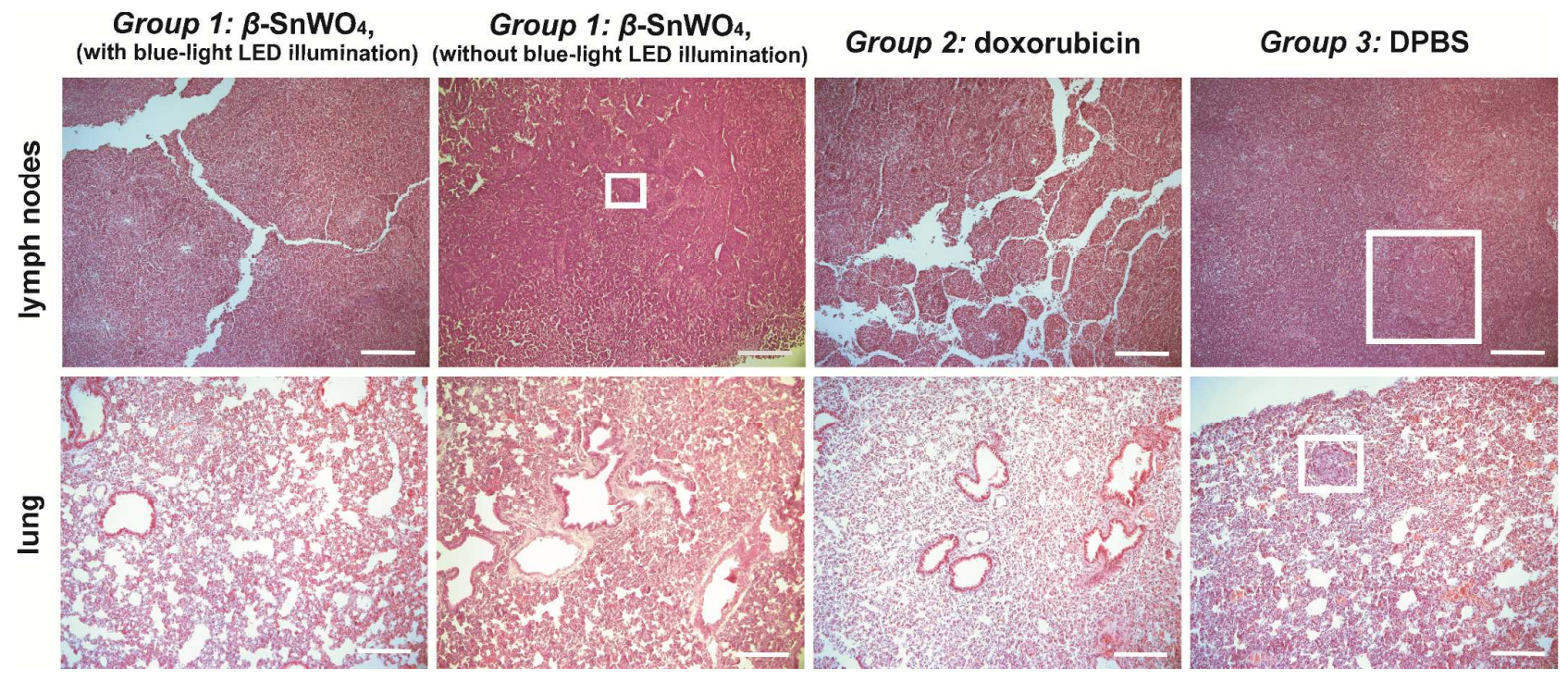

Figure S7. Effect of $\beta$-SnWO $\mathrm{O}_{4}$ nanoparticles on lymph-node metastasis (cf. main text: Figure 8): Qualitative histological analysis of lymph nodes and lungs with representative haematoxylin- and eosin-stained histological sections showing metastases for DPBS-treated mice only (see frame) (group 1, $n=6$-treated with $\beta$-SnWO $\mathrm{S}_{4}$ nanoparticles with and without blue-light LED illumination; group 2, $n=6$-treated with doxorubicin; group 3, $n=6$-treated with DPBS as described in Figure 6; scale bar: $200 \mu \mathrm{m})$. 
To our surprise, $\beta$-SnWO 4 -treated mice show an almost similar significant decrease of the tumor volume independent of the blue-light LED illumination (Figure S6). However, this finding can be ascribed to the fact that living mice cannot be kept in the dark. Consequently, continuous daylight illumination (all day long) after injection of the $\beta-\mathrm{SnWO}_{4}$ nanoparticles results in a comparable effect as short-timed (5 min), but direct and intense blue-light LED illumination (Figure S6). In fact, this is in accordance to previous in vitro studies ${ }^{1}$ showing that $\beta-\mathrm{SnWO}_{4}$ is also activated by daylight. Short-timed blue-light LED illumination is most effective for small sized tumors $\left(<2 \mathrm{~cm}^{3}\right.$, day 8 to day 18$)$ that are completely transilluminated (Figure S6). Lymph-node metastasis (Figure S7) and lymph-node volume (Figure S8) as well indicate the influence of daylight illumination on mice that were treated with $\beta$-Sn $\mathrm{WO}_{4}$ nanoparticles but not illuminated via blue-light LED. The occurrence of lymph-node metastasis (Figure S7) as well as the significantly larger ipsilateral axillary and ipsilateral inguinal lymph nodes (Figure S8), on the other hand, clearly point to the effectiveness of the blue-light LED illumination.

a) Axillary lymph nodes

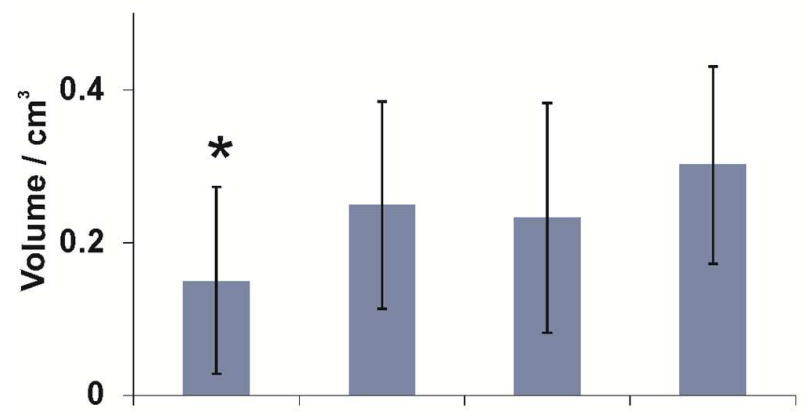

b) Inguinal lymph nodes

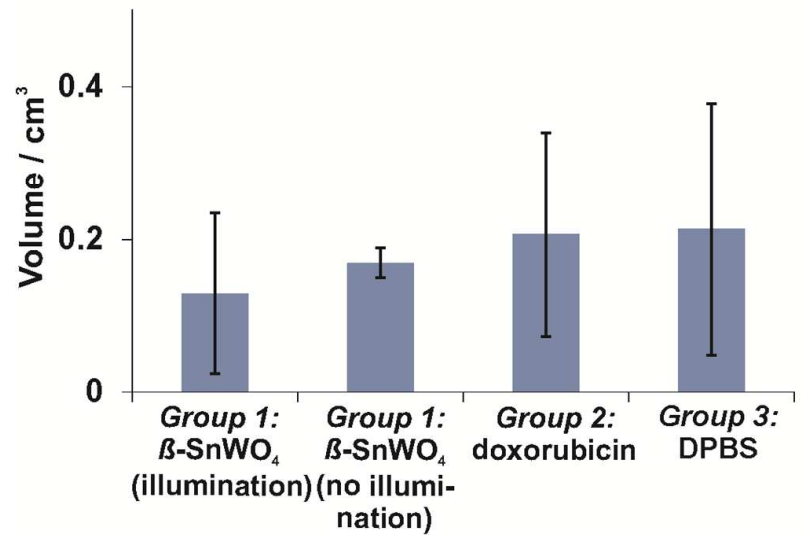

Figure S8. Effect of $\beta$-SnWO 4 nanoparticles on lymph-node volume (cf. main text: Figure 9): a) Mean volume of ipsilateral axillary lymph nodes; b) Mean volume of ipsilateral inguinal lymph nodes (Group 1, $n=6$-treated with $\beta$-SnWO $\mathrm{W}_{4}$ nanoparticles with and without blue-light LED illumination; group 2, $n=6$-treated with doxorubicin; group 3, $n=6$-treated with DPBS as described in Figure 6). The significance between the respective groups was determined according to student's $t$-test with $p<0.05$. 


\section{Blood parameters}

The blood parameters as collected for all mice on the day of sacrification are summarized in Table S1.

Table S1. The blood parameters (MV $\pm S E, n=6)$ were obtained from blood that was collected on the day of mice sacrification. Significant aberrations as compared to values obtained in the control group are shown in red and tagged with (*). Student's $t$-test served for calculation of significances (significance level $p<0.05$ ).

\begin{tabular}{|c|c|c|c|c|c|c|}
\hline & \multirow[t]{3}{*}{ Unit } & $\mathrm{SnWO}_{4}$ & $\mathrm{SnWO}_{4}$ & \multirow[t]{3}{*}{ Doxorubicin } & \multirow{3}{*}{$\begin{array}{l}\text { DPBS } \\
\text { control }\end{array}$} & \multirow[t]{3}{*}{ References $^{8-12}$} \\
\hline & & & LED & & & \\
\hline & & illumination & tion & & & \\
\hline$\alpha$-Amylase & $\mathrm{U} / \mathrm{L}$ & $2187.5 \pm 303.8$ & $2011.7 \pm 132.8$ & $1898.7 \pm 132.4$ & $1944.4 \pm 152.2$ & $2035 \pm 355$ \\
\hline Lipase & $\mathrm{U} / \mathrm{L}$ & $29.9 \pm 8.2$ & $29.4 \pm 11.4$ & $53.0 \pm 24.3^{*}$ & $25.3 \pm 3.9$ & 901 \\
\hline Fructosamines & $\mu \mathrm{mol} / \mathrm{L}$ & $194.5 \pm 13.6$ & $289.7 \pm 10.9 *$ & $171.3 \pm 31.0$ & $194.1 \pm 16.0$ & \\
\hline Triglycerides & $\mathrm{mmol} / \mathrm{L}$ & $1.6 \pm 0.2$ & $1.5 \pm 0.1$ & $1.6 \pm 0.5$ & $1.2 \pm 0.4$ & $1.15 \pm 0.40 ; 1.02 \pm 0.18 ; 2.2$ \\
\hline Cholesterol & $\mathrm{mmol} / \mathrm{L}$ & $2.0 \pm 0.2$ & $1.9 \pm 0.1$ & $2.1 \pm 0.4$ & $1.8 \pm 0.5$ & $2.31 \pm 0.41 ; 2.6$ \\
\hline Bilirubinin total & $\mu \mathrm{mol} / \mathrm{L}$ & $2.6 \pm 0.6$ & $2.4 \pm 1.8$ & $2.3 \pm 0.5$ & $3.3 \pm 3.1$ & $5.99 \pm 2.22 ; 7.2$ \\
\hline $\mathbf{A} \mathbf{P}^{3}$ & $\mathrm{U} / \mathrm{L}$ & $42.3 \pm 6.4$ & $49.3 \pm 9.9$ & $23.2 \pm 5.9 *$ & $38.8 \pm 8.1$ & $139 \pm 43 ; 129 \pm 28 ; 84$ \\
\hline GLDH $^{4}$ & $\mathrm{U} / \mathrm{L}$ & $87.3 \pm 59.9$ & $187.8 \pm 103.8$ & $28.8 \pm 12.0$ & $63.4 \pm 93.4$ & $318 \pm 222 ;$ ca. $13 \pm 3$ \\
\hline$\gamma-\mathbf{G T}^{5}$ & $\mathrm{U} / \mathrm{L}$ & $1.3 \pm 1.8$ & $1.1 \pm 0.7$ & $0.7 \pm 0.5$ & $1.5 \pm 2.3$ & \\
\hline $\operatorname{ALT}(G P T)^{6}$ & $\mathrm{U} / \mathrm{L}$ & $147.2 \pm 92.6$ & $112.4 \pm 34.0$ & $144.3 \pm 85.5$ & $85.2 \pm 36.6$ & $24 \pm 10 ; 49 \pm 5 ; 34 \pm 4 ; 61$ \\
\hline $\operatorname{AST}(G O T)^{7}$ & $\mathrm{U} / \mathrm{L}$ & $242.1 \pm 94.3$ & $449.1 \pm 267.2$ & $226.5 \pm 76.5$ & $186.8 \pm 89.5$ & $47 \pm 13 ; 150 \pm 17 ; 140 \pm 13 ; 75$ \\
\hline $\mathbf{C K}^{8}$ & $\mathrm{U} / \mathrm{L}$ & $355.5 \pm 334.3$ & $693.8 \pm 508.4$ & $348.5 \pm 104.9$ & $223.7 \pm 123.6$ & $199 \pm 167.327$ \\
\hline Proteinin total & $\mathrm{g} / \mathrm{L}$ & $54.3 \pm 3.7$ & $55.7 \pm 0.6$ & $45.7 \pm 6.9 *$ & $53.3 \pm 2.3$ & $40.1 \pm 8.9 ; 63$ \\
\hline Albumin & $\mathrm{g} / \mathrm{L}$ & $33.0 \pm 3.8$ & $33.2 \pm 1.0$ & $29.3 \pm 4.3$ & $30.6 \pm 3.4$ & 33 \\
\hline Globulines & $\mathrm{g} / \mathrm{L}$ & $23.0 \pm 1.0$ & $22.8 \pm 1.2$ & $16.6 \pm 3.9$ & $22.7 \pm 5.1$ & \\
\hline A/G-quotient & & $1.3 \pm 0.0$ & $1.3 \pm 0.0$ & $1.8 \pm 0.4 *$ & $1.1 \pm 0.2$ & \\
\hline Urea & $\mathrm{mmoL} / \mathrm{L}$ & $10.3 \pm 1.3$ & $7.1 \pm 0.6^{*}$ & $11.0 \pm 0.7$ & $12.5 \pm 3.4$ & $7.92 \pm 3.41 ; 9$ \\
\hline Creatinine & $\mu \mathrm{mol} / \mathrm{L}$ & $19.6 \pm 5.0$ & $16.8 \pm 1.3$ & $16.8 \pm 6.5$ & $16.9 \pm 3.4$ & $19.45 \pm 16.80 ; 10.2$ \\
\hline Phosphate-inorganic & $\mathrm{mmol} / \mathrm{L}$ & $4.0 \pm 1.1$ & $3.4 \pm 0.5$ & $3.4 \pm 0.9$ & $3.2 \pm 0.3$ & 2.3 \\
\hline Magnesium & $\mathrm{mmol} / \mathrm{L}$ & $1.3 \pm 0.1$ & $1.3 \pm 0.1$ & $1.1 \pm 0.2$ & $1.2 \pm 0.1$ & 1.3 \\
\hline Calcium & $\mathrm{mmol} / \mathrm{L}$ & $2.5 \pm 0.2$ & $2.6 \pm 0.2$ & $2.4 \pm 0.1^{*}$ & $2.6 \pm 0.2$ & $2.08 \pm 0.12 ; 2.4$ \\
\hline Potassium & $\mathrm{mmol} / \mathrm{L}$ & $8.9 \pm 1.4$ & $12.7 \pm 1.8$ & $8.7 \pm 1.1$ & $31.8 \pm 51.6$ & $4.40 \pm 0.96 ; 6.6$ \\
\hline Sodium & $\mathrm{mmol} / \mathrm{L}$ & $148.8 \pm 2.0$ & $148.8 \pm 2.9$ & $148.8 \pm 2.9$ & $126.1 \pm 52.6$ & $155.5 \pm 3.96 ; 179.2$ \\
\hline Iron & $\mu \mathrm{mol} / \mathrm{L}$ & $22.6 \pm 8.1$ & $30,5 \pm 1,6$ & $25.5 \pm 4.4$ & $26.6 \pm 3.6$ & 24.71 \\
\hline Erythrocytes & $\mathrm{T} / \mathrm{L}$ & $8.7 \pm 1.7$ & $8.3 \pm 0.5$ & $7.6 \pm 1.1$ & $9.0 \pm 1.4$ & \\
\hline Hematocrit & $\mathrm{L} / \mathrm{L}$ & $0.5 \pm 0.1$ & $0.4 \pm 0.0$ & $0.4 \pm 0.1$ & $0.5 \pm 0.0$ & \\
\hline Hemoglobin & $\mathrm{g} / \mathrm{L}$ & $130.8 \pm 21.9$ & $125.3 \pm 5.5$ & $117.3 \pm 14.4^{*}$ & $137.8 \pm 13.9$ & \\
\hline Leukocytes & $\mathrm{G} / \mathrm{L}$ & $77.2 \pm 28.7$ & $48.6 \pm 9.2$ & $38.7 \pm 17.4$ & $161.3 \pm 187.2$ & \\
\hline Segmented neutrophils & $\%$ & $50.7 \pm 14.3 *$ & $58.2 \pm 5.6$ & $47.2 \pm 19.4$ & $66.8 \pm 6.7$ & \\
\hline Lymphocytes & $\%$ & $30.3 \pm 10.8$ & $24.5 \pm 6.8$ & $28.2 \pm 19.7$ & $26.8 \pm 6.4$ & \\
\hline Monocytes & $\%$ & $3.3 \pm 2.4$ & $3.5 \pm 0.9$ & $1.7 \pm 1.1 *$ & $3.5 \pm 1.3$ & \\
\hline Eosinophils & $\%$ & $14.8 \pm 6.1 *$ & $9.7 \pm 1.8^{*}$ & $19.3 \pm 11.1^{*}$ & $2.8 \pm 1.2$ & \\
\hline Basophiles & $\%$ & $0.8 \pm 0.7 *$ & $1.5 \pm 1.0 *$ & $3.8 \pm 3.3^{*}$ & $0.0 \pm 0.0$ & \\
\hline
\end{tabular}




\begin{tabular}{llllll} 
Unsegmented neutrophils & $\%$ & $0.0 \pm 0.0$ & $0.0 \pm 0.0$ & $0.0 \pm 0.0$ & $0.0 \pm 0.0$ \\
Hypochromasia & & neg & neg & neg & neg \\
Anisocytosis & & neg & neg & neg & neg \\
Thrombocytes & $\mathrm{G} / \mathrm{L}$ & $225.0 \pm 56.7$ & $247.7 \pm 36.1$ & $215.7 \pm 53.9$ & $293.8 \pm 136.3$ \\
Segmented neutrophils & $\mathrm{G} / \mathrm{L}$ & $36.9 \pm 14.2$ & $28.4 \pm 6.5$ & $17.6 \pm 10.1$ & $116.4 \pm 146.6$ \\
Lymphocytes & $\mathrm{G} / \mathrm{L}$ & $25.6 \pm 17.4$ & $11.6 \pm 2.8$ & $13.4 \pm 0.175$ & $33.6 \pm 26.4$ \\
Monocytes & $\mathrm{G} / \mathrm{L}$ & $2.43 \pm 1.7$ & $3.2 \pm 1.2$ & $0.5 \pm 0.5$ & $6.6 \pm 9.8$ \\
Eosinophils & $\mathrm{G} / \mathrm{L}$ & $11.3 \pm 6.9$ & $4.8 \pm 1.5$ & $6.0 \pm 1.1$ & $4.7 \pm 5.7$ \\
Basophiles & $\mathrm{G} / \mathrm{L}$ & $0.8 \pm 0.8$ & $\mathbf{0 . 7} \pm 0.5^{*}$ & $1.3 \pm 1.1^{*}$ & $0.0 \pm 0.0$ \\
Unsegmented neutrophils & $\mathrm{G} / \mathrm{L}$ & $0.0 \pm 0.0$ & $0.0 \pm 0.0$ & $0.0 \pm 0.0$ & $0.0 \pm 0.0$ \\
\hline AP: alkaline phosphatase & \multicolumn{5}{l}{} \\
GLDH: glutamate dehydrogenase \\
$\gamma$-GT: $\gamma$-glutamyl-transferase \\
ALT (GPT): alanine-amino-transferase (also: glutamic-pyruvate transaminase) \\
AST (GOT): aspartate aminotransferase (also: glutamic oxaloacetic transaminase) \\
CK: creatine kinase
\end{tabular}

\section{Haematoxylin- and eosine- staining (progressive)}

Paraffin-embedded organs were cut into $7 \mu \mathrm{m}$ sections using a Leica RM 2155 microtome. Sections were transferred to slides and dried overnight at room temperature, and subsequently in an oven at $43{ }^{\circ} \mathrm{C}$. Sections were stained and dehydrated according to the following protocol: The sections were incubated $2 \cdot 5$ minutes with xylene, 1 minutes with $100 \%$ ethanol, $2 \cdot 1$ minutes with $95 \%$ ethanol, 1 minutes with $70 \%$ ethanol, 3 minutes with distilled water, 7 minutes with haematoxylin (filtered), 3 minutes with distilled water, and 30 seconds with eosin. The sections were now rinsed with distilled water and again incubated with an increasing gradient of ethanol $(2 \cdot 1$ minutes of $70 \%, 95 \%, 100 \%$ ethanol, respectively) and finally $2 \cdot 1$ minutes with xylene. Afterwards, sections were mounted, covered with a coverslip and dried overnight. Microscopy was performed with a Zeiss Axioskop.

\section{References}

1. Ungelenk, J.; Feldmann, C. Synthesis of Faceted $\beta-\mathrm{SnWO}_{4}$ Microcrystals with Enhanced VisibleLight Photocatalytic Properties. Chem. Commun. 2012, 48, 7838-7840.

2. Wang, C.; Ma, X.; Ye, S.; Cheng, L.; Yang, K.; Guo, L.; Li, C.; Li, Y.; Liu, Z. Protamine Functionalized Single-Walled Carbon Nanotubes for Stem Cell Labeling and in vivo Raman/Magnetic Resonance/Photoacoustic Triple-Modal Imaging. Adv. Funct. Mater. 2012, 22, 2363-2375.

3. Liscovitch, M.; Lavie, Y. Cancer Multidrug Resistance: A Review of Recent Drug Discovery Research. Drugs 2002, 5, 349-355. 
4. Nichols, J. W.; Bae, Y. H. Odyssey of a Cancer Nanoparticle: From Injection Site to Site of Action. Nano Today 2012, 7, 606-618.

5. Gabizon, A.; Tzemach, D.; Mak, L.; Bronstein, M.; Horowitz, A. T. Dose Dependency of Pharmacokinetics and Therapeutic Efficacy of PEGylated Liposomal Doxorubicin (DOXIL) in Murine Models. J. Drug Target. 2002, 10, 539-548.

6. $\quad$ Kim, E. J.; Lim, K. M.; Kim, K. Y.; Bae, O. N.; Noh, J. Y.; Chung, S. M.; Shin, S.; Yun, Y. P.; Chung, J. H. Doxorubicin-Induced Platelet Cytotoxicity: A New Contributory Factor for Doxorubicin-Mediated Thrombocytopenia. J. Thromb. Haemost. 2009, 7, 1172-1183.

7. Fleig, S. in: Medizinischen Fakultät, Charité - Universitätsmedizin Berlin, Berlin 2011.

8. Kim, M. J.; Kim, H. K. Effect of Garlic on High Fat Induced Obesity. Acta Biol. Hung. 2011, 62, 244-254.

9. Le Minh, K.; Klemm, K.; Abshagen, K.; Eipel, C.; Menger, M. D.; Vollmar, B. P. Prophylaktische wie auch therapeutische Gabe von Darbepoetin alpha wirkt anti-inflammatorisch und antiapoptotisch im Modell des akuten septischen Leberschadens der Maus. Chirurg. For. 2007, 36, 205-207.

10. Mazzaccara, C.; Labruna, G.; Cito, G.; Scarfo, M.; De Felice, M.; Pastore, L.; Sacchetti, L. AgeRelated Reference Intervals of the Main Biochemical and Hematological Parameters in C57BL/6J, 129SV/EV and C3H/HeJ Mouse Strains. PloS one 2008, 3, e3772.

11. Schledzewski, K.; Geraud, C.; Arnold, B.; Wang, S.; Grone, H. J.; Kempf, T.; Wollert, K. C.; Straub, B. K.; Schirmacher, P.; Demory, A.; et al. Deficiency of Liver Sinusoidal Scavenger Receptors Stabilin-1 and -2 in Mice Causes Glomerulofibrotic Nephropathy via Impaired Hepatic Clearance of Noxious Blood Factors. J. Clin. Invest. 2011, 121, 703-714.

12. Zur, B. in: Institut für Klinische Chemie und Laboratoriumsdiagnostik, Heinrich-Heine-Universität Düsseldorf, Düsseldorf 2005. 